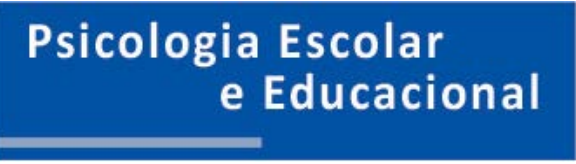

ARTIGO

DOI: http://dx.doi.org/10.1590/2175-35392021226820

Localizador - e226820

\title{
RISCO DE SUICÍDIO EM ADOLESCENTES: ESTRATÉGIAS DE PREVENÇÃO PRIMÁRIA NO CONTEXTO ESCOLAR
}

\author{
Giovana Coghetto Sganzerla ${ }^{1}(\mathbb{D}$
}

RESUMO

O presente estudo objetivou abordar estratégias de prevenção primária para o risco de suicídio em adolescentes no contexto escolar, além de investigar fatores associados, sinais de alerta e possíveis propostas de intervenção, através de uma revisão narrativa da literatura. Os materiais que serviram de referência para este estudo foram coletados a partir de bases de dados eletrônicas (SciELO, PubMed e PePSIC), livros e manuais. Identificar e compreender os fatores de risco e os sinais de alerta para o risco de suicídio em adolescentes é necessário para que se possa intervir de modo eficaz. Dentre as estratégias preventivas encontradas, estão a capacitação de professores, orientação às famílias e treinamento de habilidades sociais com os estudantes. Para que essas estratégias sejam efetivas, destacase a importância do psicólogo na composição da equipe escolar, além da necessidade de maiores investimentos para que programas de intervenção voltados à prevenção do suicídio em adolescentes sejam criados.

Palavras-chave: suicídio; prevenção; psicologia escolar

\section{Adolescent suicide risk: primary prevention strategies in the school context}

\begin{abstract}
This study aimed to discuss primary prevention strategies for the risk of suicide in adolescents in the school context, in addition to investigating associated factors, warning signs and possible intervention proposals, through a narrative review of the literature. The materials researched as reference for this study were collected from electronic databases (SciELO, PubMed and PePSIC), books and manuals. Identifying and understanding risk factors and warning signs for the risk of suicide in adolescents is necessary in order to intervene effectively. Among the preventive strategies found are the teachers' training, guidance to families and students' training in social skills. For these strategies to be effective, the importance of the psychologist in the school team is highlighted, in addition to the need for greater investments so that intervention programs aimed at preventing suicide in adolescents must be created.
\end{abstract}

Keywords: suicidal behavior; prevention; school psychology

\section{Riesgo de suicidio en adolescentes: estrategias de prevención primaria en el contexto escolar}

\section{RESUMEN}

En el presente estudio se tuvo por objetivo abordar estrategias de prevención primaria para el riesgo de suicidio en adolescentes en el contexto escolar, además de investigar factores asociados, señales de alerta y posibles propuestas de intervención, por intermedio de una revisión narrativa da literatura. Los materiales que sirvan de referencia para este estudio fueron recopilados a partir de bases de datos electrónicas (SciELO, PubMed y PePSIC), libros y manuales. Identificar y comprender los factores de riesgo y las señales de alerta para el riesgo de suicidio en adolescentes es necesario para que se pueda intervenir de modo eficaz. Entre las estrategias preventivas encontradas, están la capacitación de profesores, orientación a las familias y entrenamiento de habilidades sociales con los estudiantes. Para que estas estrategias sean efectivas, se destaca la importancia del psicólogo en la composición del equipo escolar, además de la necesidad de más inversiones para que programas de intervenciones volcados a la prevención del suicidio em adolescentes sean creados.

Palabras clave: comportamiento suicida; prevención; psicología escolar

$\overline{1}$ Pontifícia Universidade Católica do Rio Grande do Sul - Porto Alegre - RGS - Brasil. giovanasgan@gmail.com 


\section{INTRODUÇÃO}

O cenário alarmante provocado pelos elevados índices de suicídio tem constituído tal tema como um problema de saúde pública (OMS, 2000b). Mais de 800 mil pessoas morrem por suicídio anualmente, representando uma taxa de 11,4 a cada 100.000 casos de morte em todo o mundo (WHO, 2014). Entre 2011 e 2016, foram registrados 1.173 .418 casos de violência interpessoal ou autoprovocada no Sistema de Informação de Agravos de Notificação (SINAN), sendo que deste total, $15 \%$ estavam relacionados à prática de lesão autoinfligida (Ministério da Saúde, 2017). Além disso, o número de suicídios entre adolescentes vem crescendo tanto que, entre jovens de 15 a 29 anos, tornou-se a segunda principal causa de morte no mundo, denotando, assim, a necessidade de ações preventivas destinadas a esse público (WHO, 2014).

No Brasil, a taxa de mortalidade por suicídio é de 5,8 a cada 100.000 habitantes (Botega, 2015) e, de 2002 a 2012 , obteve-se um aumento de $33,6 \%$ dos casos, número maior ao crescimento da população do país nesse período (Moreira \& Bastos, 2015). Em comparação a outros países, este coeficiente de mortalidade por suicídio é relativamente baixo, sendo que o Brasil é um país populoso. No entanto, sabe-se que o nosso país ocupa a oitava posição dentre os que registram maiores números de mortes por suicídio no mundo (Botega, 2015).

Tendo em vista o aumento significativo nos índices de suicídio entre idosos (Ministério da Saúde, 2017) e a redução das taxas de crescimento populacional brasileiro nos últimos anos (IBGE, 2018; OPAS, 2008), nota-se o envelhecimento da população, o que acarreta em mais investimentos em políticas públicas destinadas aos idosos. Sabe-se, ainda, que a incidência de casos de autoextermínio tem diminuído entre idosos, mas entre jovens tem aumentado (Calixto Filho \& Zerbini, 2016). Por conta disso, pensar em ações voltadas ao público adolescente é de suma importância, considerando que o foco das intervenções governamentais atualmente se dirige à população em ascendência - os idosos.

Ademais, é importante destacar os locais de ocorrência dos casos de tentativas de suicídio e lesões autoprovocadas. Segundo o mais recente Boletim Epidemiológico divulgado pela Secretaria de Vigilância em Saúde (Ministério da Saúde, 2017), a escola aparece como cenário dessas ações, sendo sua incidência maior entre adolescentes do sexo feminino. Dessa forma, acredita-se que a escola tem um papel fundamental na prevenção e na identificação desses fatores, visto que "é o local onde são reproduzidos padrões de comportamentos e relacionamentos que podem pôr em risco a saúde dos jovens" (Baggio, Palazzo, \& Aerts, 2009, p. 143). Assim, fica evidente a necessidade de se criar estratégias de prevenção primária, secundária e terciária para o risco de suicídio no contexto escolar, uma vez que é um tema imprescindível de ser trabalhado tanto com os jovens, quanto com suas famílias e com os profissionais da Educação que atuam diretamente com esse público.

A relevância deste estudo está na urgência em se falar sobre esse tema que ainda é considerado um tabu (Calixto Filho \& Zerbini, 2016) através de uma perspectiva preventiva, atrelando a escola como uma importante aliada nesse sentido. Por conta das subnotificações e registros subestimados, estima-se que os números reais de morte por suicídio podem ser ainda maiores do que os registros oficiais do SINAN. Nesse sentido, as ações do poder público ficam restritas, pois dependem dessas notificações para promover intervenções efetivas. Além disso, sabe-se que os custos relacionados à pósvenção do suicídio são maiores em comparação aos de prevenção (DGS, 2017).

Nesse sentido, o presente artigo tem como objetivo apresentar estratégias de prevenção primária para o risco de suicídio em adolescentes na escola, além de abordar aspectos referentes ao tema, como fatores associados, sinais de alerta e, por fim, propostas de intervenção possíveis no âmbito escolar através de uma revisão narrativa da literatura.

\section{TERMINOLOGIA: DO PENSAMENTO AO COMPORTAMENTO SUICIDA}

Para um melhor entendimento a respeito do tema, faz-se necessária uma explanação sobre as diferentes nomenclaturas utilizadas. O comportamento suicida é entendido como uma ação em que um indivíduo causa lesão a si mesmo, independentemente do grau de letalidade e da real motivação por trás do ato. Concebe-se o suicídio ao longo de um continuum, que se inicia através de uma ideação suicida, ameaças de morte, gestos, tentativas de suicídio e, por fim, a consumação deste. Sendo assim, é por meio desses conceitos que se investiga a intencionalidade suicida e o grau de risco em que a pessoa se encontra (Botega, 2015).

A ideação suicida se refere a pensamentos de autodestruição e o desejo de dar fim à própria vida. Ter esse tipo de pensamento esporadicamente no período da adolescência pode ser considerado normal, visto que é uma fase de grandes mudanças. Por outro lado, dependendo da intensidade e da frequência desses pensamentos, eles podem se caracterizar como um sinal de alerta para o risco de suicídio se o adolescente acreditar que a realização deles é a única solução para os seus problemas (Moreira \& Bastos, 2015). De acordo com Calixto Filho e Zerbini (2016), a ideação suicida é prevalente ao longo da vida, acometendo cerca de $17 \%$ da população brasileira, seguida da estruturação de um plano, cuja incidência é de $4,8 \%$.

O planejamento envolve o método específico a ser utilizado para consumar o suicídio (Claumann, Pinto, 
Silva, \& Pelegrini, 2017). A existência de um plano caracteriza o indivíduo com grau elevado de risco, pois além da intenção de morte, ele já tem um local e provavelmente uma data definida para cometer suicídio, assim como o meio (arma de fogo, enforcamento, medicamento ou veneno, por exemplo) e já tomou algumas providências prévias diante de sua ideia de morte (Botega, 2015). Dessa forma, após o plano, vem a tentativa ou o parassuicídio.

O termo parassuicídio, segundo Piñero, Blasco, Baca-Garcia e Diaz (2002, citado por Araújo, Vieira, \& Coutinho, 2010), refere-se às condutas cuja intenção não é a de tirar a própria vida, mas de tentar modificar o contexto no qual o indivíduo está inserido. Já a tentativa de suicídio se diferencia pela intencionalidade, que é a de morrer, e pelo grau de letalidade do método. $A$ consumação desses atos caracteriza o suicídio.

A etimologia da palavra suicídio deriva do latim que significa ação de matar (caedes) a si mesmo (sui) (Correa \& Barreto, 2006, citado por Moreira \& Bastos, 2015). Assim como na tentativa, na consumação há o desejo de morrer, caracterizando-se como um ato voluntário que provoca a própria morte (Vieira, 2008, citado por Araújo et al., 2010).

\section{IDEAÇÃO SUICIDA EM ADOLESCENTES: FATORES ASSOCIADOS}

Através da identificação dos fatores associados ao suicídio, pode-se planejar ações preventivas mais precisas no âmbito da escola. Dentre esses fatores, estão o pequeno número de amigos e as relações agressivas que o indivíduo estabelece com esses (Souza et al., 2010). A escola, por sua vez, tem uma função significativa na vida das crianças e dos adolescentes pois é conhecida por promover saúde e bem-estar a eles (Haavet, Dalen, \& Straand, 2005), principalmente por ser um dos primeiros ambientes em que a criança aprende a socializar. Quando não há um sentimento de pertença ou quando um indivíduo é excluído de um grupo, as chances de desenvolver sintomas depressivos são aumentadas (Barros, Coutinho, Araújo, \& Castanha, 2006; Forlim, Stelko-Pereira, \& Williams, 2014). Ademais, ser vítima de bullying também prediz maior propensão ao desenvolvimento de distúrbios de ordem psicológica (Kumpulainen et al., 1998). Em relação à agressividade presente nas relações, sabe-se que a variável do comportamento impulsivo-agressivo está presente em muitos dos casos de suicídio consumado (Turecki, 1999) e que pode estar associada à própria fase desenvolvimental da adolescência (Crestani, 2016).

Outro fator fortemente associado à ideação suicida em adolescentes é a existência de transtornos psiquiátricos (Souza et al., 2010; Moreira \& Bastos, 2015; Botega, Barros, De Oliveira, Dalgalarrondo, \& Marín-Léon, 2004). A condição clínica de um indivíduo portador de algum transtorno mental pode afetar sua vida nas esferas social, profissional e acadêmica. Assim, ele poderá apresentar: maior dificuldade em se adaptar à sociedade; sintomas como ansiedade, raiva e frustração e piora na qualidade de vida. Esses fatores, aliados a um diagnóstico não fidedigno e a um tratamento inapropriado, podem agravar o risco de morte de um indivíduo que possui ideação suicida. Dentre os transtornos mentais mais comuns associados ao suicídio estão o Transtorno Depressivo Maior, o Transtorno Bipolar, a Dependência de Álcool e Outras Drogas, Esquizofrenia e Transtornos de Personalidade que incluam características como impulsividade, agressividade e labilidade (Botega, 2015). Além disso, sintomas como tristeza, solidão e baixa autoestima estão relacionados ao planejamento suicida, independentemente da existência de um transtorno (Baggio et al., 2009; Souza et al., 2010).

Problemas comportamentais, uso de substâncias, histórico de abuso e exposição à violência, relacionamento familiar disfuncional, assim como pobreza de habilidades para solucionar problemas também são fatores associados ao suicídio em adolescentes (Claumann et al., 2018; Baggio et al., 2009; Moreira \& Bastos, 2015; Souza et al., 2010; Botega et al., 2004). Além disso, conhecer alguém que já tenha cometido suicídio é outro fator importante, sendo explicado pelo Efeito Werther, que sugere que o comportamento suicida pode ser aprendido através de modelagem (Almeida, 2000). Outro fator associado que merece ressalva é a dificuldade em lidar com a imagem corporal. Deve-se ter em mente que a adolescência é considerada um período conturbado devido às inúmeras mudanças atreladas à puberdade. Assim, é importante identificar esse fator como uma característica própria desse período, desde que não traga riscos à vida do indivíduo, uma vez que essa condição pode influenciar a susceptibilidade a transtornos mentais - como Transtorno Depressivo Maior ou Transtornos Alimentares (Claumann et al., 2017; Baggio et al., 2009).

\section{SINAIS DE ALERTA PARA O RISCO DE SUICÍDIO EM ADOLESCENTES}

Identificar e compreender os sinais de alerta para o risco de suicídio em adolescentes é necessário para que se possa intervir de modo eficaz. Apesar disso, é importante saber diferenciar reações consideradas normais à adolescência de sinais de alerta. A dificuldade está em identificar esta diferença, pois muitos desses sinais não são específicos e podem aparecer através de transtornos mentais que, por sua vez, costumam ter início no período da adolescência (Botega, 2015).

Hawton, Saunders e O'Connor (2012, p. 2375) fazem referência a catorze principais sinais de que o adolescente pode estar em risco de suicídio. Dentre eles, estão as "mudanças marcantes na personalidade 
ou nos hábitos" assim como a manifestação de "comportamento ansioso, agitado ou deprimido". Sempre que se notarem mudanças significativas no indivíduo, é preciso prestar atenção. Alguns comportamentos que podem ser considerados atípicos, para um adolescente pode ser normal tendo em vista que essa é uma fase de muitas contradições, ambivalências e conflitos (Moreira \& Bastos, 2015).

Pensando no contexto escolar, um terceiro fator que aparece como sinal de alerta é a piora no desempenho escolar e o desinteresse em atividades que o indivíduo costumava fazer. Sinais como problemas de comportamento na escola, declínio geral nas notas e excesso de faltas são indicativos de que algo errado pode estar acontecendo. Por isso, deve-se dar maior atenção para qualquer mudança súbita no comportamento que interfira no desempenho escolar do estudante (OMS, 2000a).

Outro fator importante mencionado pelos autores é o "afastamento da família e dos amigos". Quando ocorre esse desligamento em relação aos familiares, pode-se entender esse movimento como uma fase esperada do desenvolvimento psíquico do adolescente, em que o investimento se dá em novos objetos que não mais os pais (Macedo, Azevedo, \& Castan, 2012). Por outro lado, quando há um afastamento não só em relação à família, mas também aos amigos, tem-se um indicador de isolamento social, que pode estar associado a algum transtorno de ordem psicológica e, portanto, merece maior atenção. Somado a isso, tem-se a perda de interesse por atividades que costumava gostar, desesperança, comentários negativos em relação ao futuro e mudanças no padrão de sono que também podem configurar indícios de transtornos mentais, podendo indicar risco aumentado para o suicídio (Hawton, Saunders \& O'Connor, 2012).

Além disso, fatores como "descuido com a aparência", alteração significativa do peso e "comentários autodepreciativos persistentes" (Botega, 2015, p. 157) podem caracterizar-se como sinais de risco. Tendo em vista que é um período em que o adolescente tem uma preocupação em ser aceito pelo seu grupo, o desinteresse pela aparência aparece na contramão de um comportamento típico dessa fase. Ademais, perda ou ganho de peso também podem denotar uma preocupação relacionada à imagem corporal, ao mesmo tempo que podem indicar a existência de um transtorno.

A manifestação clara ou velada do desejo de morrer, assim como fazer comentários que demonstrem um interesse pela temática - falar sobre pessoas que morreram, doar pertences valiosos e dar demais avisos - sinalizam o risco de suicídio. Nesse sentido, cabe ressaltar alguns mitos em relação ao assunto que, por vezes, são desconsiderados pela população em geral.

O primeiro deles, mencionado pela Organização Mundial da Saúde, é: "Pessoas que falam sobre suicídio não têm intenção de fazê-lo" (WHO, 2014, p. 15). Deve-se dar total atenção a falas como "eu quero sumir" ou "eu quero morrer", independente do contexto em que elas forem expressadas. Nesse sentido, é preciso ter em mente que, quando um indivíduo expõe um assunto delicado como esse, é porque ele pode estar precisando compartilhar seu sofrimento com alguém com a intenção de pedir ajuda. Outro grande engano em relação ao tema é o discurso de que a maioria dos casos de suicídio acontece sem avisos prévios e servem apenas para chamar a atenção. Como referido anteriormente, existem diversos sinais de alerta, muitas vezes não tão claros, de que uma pessoa está pensando em tirar a própria vida. Por isso, é importante não desvalorizar esses sinais e investigar a sua veracidade, visto que estão chamando a atenção para um problema sério pelo qual a pessoa pode estar passando (WHO, 2014).

Outro enunciado bastante comum sobre o tema é: "Um suicida está determinado a morrer" (WHO, 2014, p. 43). Uma das principais características de uma pessoa que possui ideação suicida é a ambivalência, que se caracteriza pela presença de sentimentos contraditórios. Uma pessoa pode ter um plano, pode estar decidida a cometer suicídio e ao mesmo tempo agir de modo a pedir ajuda a outras pessoas (Leenaars, 2010). Assim sendo, diante de situações como essa, é necessário prestar suporte emocional ao indivíduo.

Por fim, é importante ressaltar um dos maiores mitos em relação ao tema: "Falar sobre suicídio é uma má ideia e pode ser interpretado como encorajamento" (WHO, 2014, p. 65). Sabe-se que existe um estigma que impede ou dificulta a discussão sobre o tema em determinados contextos. No entanto, perguntar sobre essa intenção a alguém que esteja apresentando sinais de risco não é sinônimo de encorajamento, mas sim de sensibilidade e empatia. A atitude de uma pessoa demonstrar se importar com a outra pode servir de incentivo para desabafar sobre o que está acontecendo. Dessa forma, é importante que o profissional que tomar conhecimento da ideação suicida de uma pessoa esteja preparado para administrar a situação. Para isso, serão abordadas estratégias de prevenção para o manejo do risco de suicídio no contexto escolar.

\section{ESTRATÉGIAS DE PREVENÇÃO NA ESCOLA}

A prevenção ao suicídio pode ser classificada como universal, seletiva ou específica. A prevenção universal ou primária é aquela que objetiva reduzir a ocorrência de novos casos por meio de ações educativas; a seletiva ou secundária é destinada a grupos em situação de risco; e a específica ou terciária concentra-se em indivíduos que possuem ideação suicida e/ou planejamento (Conte et al., 2012). Diversos programas de intervenção têm sido propostos visando à prevenção do suicídio nos últimos anos (Conte et al., 2017). Entretanto, tratando-se do âmbito escolar, sabe-se que alguns grupos de risco 
não estão sendo atingidos por essas intervenções, pois a inclusão obrigatória do psicólogo nas escolas ainda é recente no Brasil.

Ao propor estratégias de prevenção primária na escola, é preciso compreender quais fatores estão associados ao risco de suicídio para que se possa intervir de forma eficaz. Como mencionado nas seções anteriores, a relação agressiva com os amigos, a comunicação disfuncional com a família, a presença de problemas comportamentais e a dificuldade em solucionar problemas aparecem como alguns desses fatores. A promoção de saúde na escola se dá, por exemplo, por meio de ações voltadas ao desenvolvimento de habilidades sociais. Sabe-se que um repertório social empobrecido pode acarretar problemas de ordem psicológica na infância e na adolescência. Assim, a competência social aparece como um indicador preciso de ajustamento psicossocial e necessário para o desenvolvimento pois aumenta a capacidade de um indivíduo para lidar com situações adversas e estressantes do cotidiano (Del Prette \& Del Prette, 2013).

Sendo assim, é importante salientar que trabalhar a competência social na escola desde a infância pode ajudar as crianças a desenvolverem habilidades de comunicação e resolução de problemas para que a trajetória desenvolvimental se torne satisfatória. Além disso, a agressividade na infância pode estar relacionada a um padrão sociocognitivo que se expressa através de falhas na identificação ou interpretação de sinais relevantes do ambiente; de tendências a atribuir intenções hostis à pessoa que está falando; dificuldade em elaborar soluções eficazes frente a demandas sociais; déficits no que se refere ao desempenho social; e de uma avaliação egocêntrica em relação aos demais, podendo trazer reflexos na adolescência (Del Prette \& Del Prette, 2013).

Somado a isso, está o fator da quantidade de amigos que adolescentes em risco de suicídio apresentam. Um estudo realizado nos Estados Unidos demonstrou que $12 \%$ das crianças possuíam apenas um amigo, $6 \%$ nenhum e de 5 a $15 \%$ das crianças apresentavam problemas graves de relacionamento interpessoal (Matson, Sevin, \& Box, 1995, citado por Del Prette \& Del Prette, 2013). Criar amizades e mantê-las depende de inúmeras condições, dentre elas a competência em habilidades sociais específicas. Por outro lado, a falta de assertividade, cooperação e empatia, pouco contato visual ou sorrisos, dificuldade em demonstrar interesse pelo outro, assim como atitudes agressivas, hiperatividade, negativismo, hostilização e excesso de críticas em relação ao outro podem prejudicar ainda mais neste sentido (Del Prette \& Del Prette, 2013).

Uma das formas de treinar habilidades sociais no contexto escolar é a partir de vivências realizadas em grupo que tenham a finalidade de promover a coesão grupal. A partir do momento em que se cria uma at- mosfera de conhecimento mútuo, é possível verificar a aquisição ou o aprimoramento de habilidades importantes para o convívio social e para a amizade (Del Prette \& Del Prette, 2013). Dessa forma, é importante ressaltar, apenas, que essas dinâmicas não devem provocar uma exposição desnecessária por parte de quem participa, pois isso pode mobilizar algumas pessoas de tal forma que o grupo possa não conseguir conter os sentimentos que emergirem. Assim, deve-se pensar quais as melhores estratégias para que se atinja determinado objetivo sem que a atividade se torne um disparador.

Outros fatores que aparecem como determinantes para o risco de suicídio em adolescentes são o histórico de abuso sexual, exposição à violência e dificuldade em lidar com a imagem corporal. A escola é o cenário ideal para que se possa discutir diferentes assuntos que abordem a temática da educação sexual no que se refere desde a violência até aspectos desenvolvimentais típicos da adolescência. A exemplo disso tem-se a taxa de notificações de violência doméstica e sexual que, em crianças/adolescentes de 10 a 19 anos, é de 25,4\% (Assis, Avanci, Pesce, Pires, \& Gomes, 2012). Dados como esse denotam a necessidade em orientar os estudantes e suas famílias para que possam identificar situações de abuso/violência. Ademais, no que tange à imagem corporal, deve-se considerar a relevância de informar os adolescentes quanto ao período do ciclo vital pelo qual estão passando. A dificuldade em relação à aceitação do próprio corpo e a forma como esses lidam com isso está estritamente relacionada a essa etapa, mas é necessário que a escola ofereça estas informações relativas à puberdade aos estudantes para que eles possam, além de conhecer o próprio corpo, compreendê-lo. Vale ressaltar que, conforme os Parâmetros Curriculares Nacionais, a escola deve servir como fonte de informação, sendo palco de discussões sobre diferentes questões consideradas tabus, com o intuito de preencher as lacunas existentes sobre temas que o adolescente tem interesse a partir do ponto de vista científico (Moreira, 2011).

Agregado a isso, tem-se a problemática em relação ao uso de álcool e outras drogas, fator encontrado em um a cada quatro pacientes suicidas (OMS, 2000a). A adolescência é uma das fases mais propícias para o início do consumo de drogas lícitas e ilícitas, incluindo-se o álcool, tendo em vista que é um período em que a curiosidade por novas experiências é aumentada. Dessa forma, como estratégia de prevenção, indica-se a orientação sobre as consequências e prejuízos à saúde decorrentes do uso e abuso de substâncias. Palestras, pesquisas sobre o tema, leituras didáticas e reflexões inseridas no plano de ensino de algumas disciplinas podem ajudar os adolescentes a visualizarem a temática sob outra perspectiva e adotarem uma postura mais cautelosa em relação a essa fase (Façanha, Erse, Simões, Amélia, \& Santos, 2010). 
Falar em prevenção ao suicídio é pensar em diferentes artifícios que englobem uma soma de fatores que estão associados à consumação do ato e considerar que esse não é um caminho de via única. Sendo assim, promover e valorizar a vida em um contexto como o da escola mostra-se a melhor alternativa. Para isso, é preciso não só direcionar estratégias aos estudantes, mas também envolver os educadores nessa missão tornando-os agentes de mudança, tendo em vista o trabalho em equipe e multidisciplinar (OMS, 2000a).

A instrumentalização daqueles que estão diariamente mais próximos aos estudantes torna-se mais eficaz, pois facilita a identificação de sinais de alerta para o risco de suicídio. Logo, indica-se a capacitação de professores por parte de profissionais da saúde mental para que, após a identificação, possa-se acionar os profissionais competentes (Baggio et al., 2009). O conhecimento sobre transtornos mentais e seus sintomas, além de todos os outros sinais discutidos anteriormente, pode auxiliar os professores a compreenderem melhor a situação pela qual um estudante está passando - falta de interesse em atividades habituais, declínio geral nas notas, mau comportamento em sala de aula e excesso de faltas, por exemplo (OMS, 2000a).

Nesse sentido, faz-se necessária, também, uma breve instrução a respeito do manejo quando o risco já está instaurado. Em um primeiro momento, deve-se considerar um local apropriado para uma conversa; nunca conversar nos corredores ou em sala de aula sobre assuntos pessoais que possam mobilizar a pessoa. Após isso, deve-se ouvir atenta e empaticamente o que a pessoa em risco tem a dizer; não é aconselhado interromper com frequência, nem ficar chocado ou muito emocionado; pelo contrário, deve-se expressar mensagens não-verbais de aceitação e respeito aos sentimentos da pessoa, conduzindo a conversa de modo cuidadoso e afetuoso (OMS, 2000b). Esses passos são recomendados a profissionais da saúde mental que atuem em espaços educativos pois, dependendo dos rumos da conversa, saberão quais as melhores medidas a serem tomadas. Todavia, é necessário entender que esse tipo de abordagem não se configura como clínica, considerando que esse não é o papel do psicólogo escolar ou do orientador educacional. Trata-se apenas de um protocolo que deve ser seguido diante de casos de risco à integridade física de um indivíduo em qualquer contexto.

Portanto, é imprescindível o cuidado em relação à saúde mental da equipe escolar como um todo. Conviver e manejar situações como essas podem sensibilizar os funcionários e, por isso, deve-se prestar suporte a estes sempre que necessário. Além disso, tratando-se de manejo, disponibilizar acesso à informação quanto aos serviços da rede de saúde mental torna-se indispensável (OMS, 2000a).
Em relação às famílias, o papel do psicólogo deve ser o de orientação. A partir do risco detectado, deve-se acionar os familiares do estudante e fazer os encaminhamentos necessários. Sabe-se, porém, que em alguns casos as famílias não seguem as instruções que foram recomendadas pelo profissional. Por isso, é importante que todos os encontros com familiares ou responsáveis legais sejam registrados e que esses possam assinar um termo afirmando estarem cientes de toda e qualquer situação de risco detectada e informada pela escola. Dessa forma, a escola e os profissionais que nela atuam estarão seguros de que seu papel foi cumprido, apesar da limitação de ação em torno de cada caso. Ademais, pensando-se em prevenção primária, é pertinente que se elaborem demais estratégias de orientação às famílias com temas referentes à saúde mental, desde a distribuição de material para leitura até ações como palestras ou até, se possível, grupos permanentes de estudo e reflexão para aprofundar o debate sobre esses assuntos que merecem maior atenção.

\section{CONSIDERAÇÕES FINAIS}

Como já referenciado em outros estudos, há a necessidade de maiores investimentos para que programas de intervenção específicos voltados à prevenção do suicídio em adolescentes sejam criados (Moreira \& Bastos, 2015; Feijó \& Oliveira, 2001; Baggio et al., 2009). Nota-se, assim, a importância da presença do psicólogo na composição da equipe escolar, recentemente garantida por Lei (Lei no 13.935, 2019). A promoção de saúde nesse contexto depende da atuação de profissionais que tenham conhecimento teórico e prático em saúde mental para que a realidade escolar possa ser mudada.

Para além do trabalho no ambiente educacional, os efeitos dessas estratégias devem ter impacto social, ultrapassando os muros da escola. A comunidade escolar como um todo, sejam estudantes, corpo docente, famílias e demais membros da sociedade, devem desfrutar dos benefícios a curto e longo prazo das ações que visam à prevenção do suicídio, mas que repercutem em diversos âmbitos no que diz respeito à saúde e qualidade de vida, como discorrido ao longo desse artigo.

Cabe destacar, portanto, o papel que a própria sociedade tem na vida de cada sujeito e, neste caso, em relação à morte em si, ao passo em que vivemos em um contexto adoecido e adoecedor ao mesmo tempo. Diversas características hoje presentes na sociedade - como individualismo, imediatismo, baixa tolerância à frustração - reforçam a necessidade desse tipo de trabalho, como bem elucidado em um estudo de Nilson Berenchtein Netto (Berenchtein Netto, 2007), que entende que, ao compreendermos o suicídio a partir de uma visão psicossocial, tornamo-nos, assim, mais preparados para intervir com eficácia. Por fim, entendendo o suicídio como uma questão de saúde pública, é preciso que, além de garantir a prevenção no ambiente 
escolar, políticas públicas abranjam esse tema como parte de suas ações em prevenção e promoção de saúde à população em geral, considerando o conceito amplo de saúde hoje vigente.

\section{REFERÊNCIAS}

Almeida, A. F. (2000). Efeito de Werther. Análise Psicológica, 18(1), 37-51.

Araújo, L. C.; Vieira, K. F. L.; Coutinho, M. P. L. (2010). Ideação suicida na adolescência: um enfoque psicossociológico no contexto do ensino médio. Psico-USF, 15(1), 47-57. https:// dx.doi.org/10.1590/S1413-82712010000100006

Assis, S. G.; Avanci, J. Q.; Pesce, R. P.; Pires, T. O.; Gomes, D. L. (2012). Notificações de violência doméstica, sexual e outras violências contra crianças no Brasil. Ciência \& Saúde Coletiva, 17(9), 2305-2317.

Baggio, L.; Palazzo, L. S.; Aerts, D. R. G. C. (2009). Planejamento suicida entre adolescentes escolares: prevalência e fatores associados. Cadernos de Saúde Pública, 25(1), 142-150.

Barros, A. P. R.; Coutinho, M. P. L.; Araújo, L. F.; Castanha, A.R. (2006). As representações sociais da depressão em adolescentes no contexto do ensino médio. Estudos em Psicologia, 23(1), 19-28. https://dx.doi.org/10.1590/S0103166X2006000100003

Berenchtein Netto, N. (2007). Suicídio: uma análise psicossocial a partir do materialismo histórico dialético (Dissertação de mestrado). Pontifica Universidade Católica, São Paulo. Recuperado de https://sapientia.pucsp.br/bitstream/ handle/17213/1/Nilson\%20Berenchtein\%20Netto.pdf

Botega, N. J. (2015). Crise Suicida: avaliação e manejo. Porto Alegre: Artmed.

Botega, N. J; Barros, M. B. A.; De Oliveira, H. B.; Dalgalarrondo, P.; Marín-Leon, L. (2004). Suicidal behavior in the community: prevalence and factors associated with suicidal ideation. Brazilian Journal of Psychiatry, 27(1), 45-53.

Lei no 13.935, de 11 de dezembro de 2019. Dispõe sobre a prestação de serviços de psicologia e de serviço social nas redes públicas de educação básica. Diário Oficial da República Federativa do Brasil. Recuperado de http:// www.planalto.gov.br/ccivil_03/_ato2019-2022/2019/lei/ L13935.htm

Calixto Filho, M.; Zerbini, T. (2016). Epidemiologia do suicídio no Brasil entre os anos de 2000 e 2010. Saúde, Ética \& Justiça, 21(2), 45-51. https://doi.org/10.11606/issn.23172770.v21i2p45-51

Claumann, G. S.; Pinto, A. A.; Silva, D. A. S.; Pelegrini, A. (2018). Prevalência de pensamentos e comportamentos suicidas e associação com a insatisfação corporal em adolescentes. Jornal Brasileiro de Psiquiatria, 67(1), 3-9. http://dx.doi. org/10.1590/0047-2085000000177

Conte, M.; Meneghel, S. N.; Trindade, A. G.; Ceccon, R. F.; Hesler, L. Z.; Cruz, C. W.; Soares, R.; Pereira, S.; Jesus, I. (2012). Programa de Prevenção ao Suicídio: estudo de caso em um município do sul do Brasil. Ciência \& Saúde Coletiva, 17(8), 2017-2026.
Crestani, Ir. A. (2016). Adolescência: tentando compreender o que é difícil entender. Porto Alegre: EdiPUCRS.

Del Prette, Z. A. P.; Del Prette, A. (2013). Psicologia das habilidades sociais na infância. Petrópolis: Editora Vozes.

Direção Geral da Saúde [DGS]. (2017). Programa Nacional de Saúde Mental: Plano Nacional de Prevenção do Suicídio 2013/2017. Portugal: DGS. Recuperado de: https://www. dgs.pt/documentos-e-publicacoes/plano-nacional-deprevencao-do-suicido-20132017-pdf.aspx

Façanha, J. D. N.; Erse, M. P. Q. A.; Simões, R. M. P.; Amélia, L.; Santos, J. C. (2010). Prevenção do suicídio em adolescentes: programa de intervenção Believe. Revista Eletrônica Saúde Mental Álcool e Drogas, 6(1), 1-16.

Feijó, R. B.; De Oliveira, E. A. (2001). Comportamento de risco na adolescência. Jornal de Pediatria, 77(2), 125-134.

Forlim, B. G.; Stelko-Pereira, A. C.; Williams, L. C. A. (2014). Relação entre bullying e sintomas depressivos em estudantes do ensino fundamental. Estudos em Psicologia, 31(3), 367-375. https://dx.doi.org/10.1590/0103$166 \times 2014000300005$

Haavet, O. R.; Dalen, I.; Straand, J. (2005). Depressive symptoms in adolescent pupils are heavly influenced by the school they go to: a study of 10th grade pupils in Oslo, Norway. European Journal of Public Health, 16(4), 400-404.

Hawton, K.; Saunders, K. E. A.; O'Connor, R. O. (2012). Selfharm and suicide in adolescents. The Lancet, 379, 23732382. doi: 10.1016/S0140-6736(12)60322-5.

Instituto Brasileiro de Geografia Estatística [IBGE]. (2018). Projeção da população. Brasil: IBGE. Recuperado em 21 de junho de 2019, de: https://www.ibge.gov.br/apps/ populacao/projecao//index.html

Kumpulainen, K.; Räsänen, E.; Henttonen, I.; Almqvist, F.; Kresanov, K.; Linna, S. L.; Moilanen, I.; ... Tamminen, T. (1998). Bullying and psychiatric symptoms among elementary school-age children. Child Abuse Neglect, 22(7), 705-717. doi: 10.1016/s0145-2134(98)00049-0.

Leenaars, A. A. (2010). Edwin S. Shneidman on Suicide. Suicidology Online, 1, 5-18. https://www.semanticscholar. org/paper/Edwin-S.-Shneidman-on-suicide-Leenaars/2cdb 6874098bc3d38dc64032ce3a4fb8c9cf8d5c\#paper-header

Macedo, M. M. K.; Azevedo, B. H.; Castan, J. U. (2012). Adolescência e Psicanálise. In: M. M. K. Macedo (Ed.), Adolescência e Psicanálise: interseç̧ões possíveis (Vol. 2, pp. 15-54). Porto Alegre: EdiPUCRS.

Ministério da Saúde (2017). Suicídio: saber, agir e prevenir, 48(30). SUS: Ministério da Saúde. Recuperado de: http://portalarquivos2.saude.gov.br/images/pdf/2017/ setembro/21/2017-025-Perfil-epidemiologico-dastentativas-e-obitos-por-suicidio-no-Brasil-e-a-rede-deatencao-a-saude.pdf

Moreira, L. M. A. (2011). A educação sexual como ação inclusiva. In: L. M. A. Moreira (Ed.), Algumas abordagens da educação sexual na deficiência intelectual. (Vol. 3, pp. 23-27). Salvador: EDUFBA. 
Moreira, L. C. de O.; Bastos, P. R. H. de O. (2015). Prevalência e fatores associados à ideação suicida na adolescência: revisão de literatura. Psicologia Escolar e Educacional, 19(3), 445-453. https://dx.doi.org/10.1590/21753539/2015/0193857

Organização Mundial da Saúde (2000a). Prevenção do suicídio: manual para professores e educadores. Genebra: OMS. Recuperado de https://www.who.int/mental_health/ prevention/suicide/en/suicideprev_educ_port.pdf

Organização Mundial da Saúde. (2000b). Prevenção do suicídio: um manual para profissionais da saúde em atenção primária. Genebra: OMS. Recuperado de https:// www.who.int/mental_health/prevention/suicide/en/ suicideprev_phc_port.pdf

Organização Pan-Americana da Saúde (2008). Indicadores básicos para a saúde no Brasil: conceitos e aplicações. Brasília: OPAS. Recuperado de https://www.paho.org/bra/ dmdocuments/indicadores.pdf

Souza, L. D. M.; Ores, L.; De Oliveira, G. T.; Cruzeiro, A. L. S.; Silva, R. A.; Pinheiro, R. T.; Horta, B. L. (2010). Ideação suicida na adolescência: prevalência e fatores associados. Jornal Brasileiro de Psiquiatria, 59(4), 286-292. http:// dx.doi.org/10.1590/S0047-20852010000400004

Turecki, G. (1999). O suicídio e sua relação com o comportamento impulsivo-agressivo. Brazilian Journal of Psychiatry, 21(2), 18-22. http://dx.doi.org/10.1590/S151644461999000600006

World Health Organization (2014). Preventing suicide: $a$ global imperative. Luxemburgo, WHO. Recuperado de: https://www.who.int/mental_health/suicide-prevention/ world_report_2014/en/

Recebido: 30 de julho de 2019 Aprovado: 20 de março de 2021 